\title{
Kissing aneurysms of the distal anterior cerebral artery: A case report and literature review
}

\author{
CHUAN-YI FU ${ }^{1}$, JIAN-LONG CHEN ${ }^{1}$, ZHAO-HUI LIU ${ }^{1}$, PENG-CHENG WANG ${ }^{1}$, \\ CHUAN-ZHI DUAN ${ }^{2}$ and JIAN-NONG ZHAO ${ }^{1}$
}

\begin{abstract}
${ }^{1}$ Department of Neurosurgery, The National Key Clinic Specialty, Hainan General Hospital, Hainan Clinical Medicine Research Institution, Haikou, Hainan 570311; ${ }^{2}$ Department of Neurosurgery, The National Key Clinic Specialty, The Engineering Technology Research Center of Education Ministry of China, Guangdong Provincial Key Laboratory on Brain Function Repair and Regeneration, Zhujiang Hospital, Southern Medical University, Guangzhou, Guangdong 510282, P.R. China
\end{abstract}

Received March 2, 2017; Accepted August 1, 2017

DOI: $10.3892 /$ etm.2018.5854

\begin{abstract}
Intracranial 'kissing' aneurysms are rare types of multiple aneurysms referring to two adjacent aneurysms arising from identical or different arteries with separate origins and partially adherent walls. The present study reported a 54-year-old female patient, who was identified with a 'kissing' aneurysm in the A3 segment of the bilateral anterior cerebral arteries, as demonstrated by head computed tomography and emergency cerebral digital subtraction angiography analysis. In total, 12 days following the clipping of the aneurysms, the patient was discharged with a Modified Rankin Scale $=0$ and recovered well with no neurological deficits. Based on previous literature, it was indicated that the majority of patients with 'kissing' aneurysm have a good prognosis and the cure rate is as high as $\mathbf{9 6 . 8 \%}$. However, the recovery rate may not be that high as the sample size is not large enough to thoroughly demonstrate the complete prognosis of 'kissing' aneurysms.
\end{abstract}

\section{Introduction}

'Kissing' aneurysms were initially described and defined by Jefferson (1). They are special types of multiple aneurysms defined as two adjacent aneurysms arising from identical or different arteries with separate origins and partially adherent walls (1). Cases of 'Kissing' aneurysms make up $<1 \%$ of all intracranial aneurysms (2). Due to 'kissing' aneurysms occurring in arteries sharing adherent walls, they may be misdiagnosed as a single aneurysm, resulting in patients undergoing the wrong treatment, which may lead to the

Correspondence to: Dr Jian-Nong Zhao, Department of Neurosurgery, The National Key Clinic Specialty, Hainan General Hospital, Hainan Clinical Medicine Research Institution, 19 Huaxiu Road, Haikou, Hainan 570311, P.R. China

E-mail: zhaojiannong421@163.com

Key words: kissing aneurysms, distal anterior cerebral artery, prognosis, case report subsequent rupture of the aneurysms (2). Fortunately, $96.8 \%$ of reported cases had a favourable prognosis with only one case having an unknown prognosis (Table I). 'Kissing' aneurysms are commonly located in the proximal of the internal carotid artery following fenestration deformity (3). Notably, 'kissing' aneurysms of bilateral distal anterior cerebral arteries (DACAs) are rare and, to date, only 3 cases of DACA 'kissing' aneurysms have been reported prior to the current case $(2,4,5)$.

The present study reported a rare instance of DACA 'kissing' aneurysms, which were clipped. To the best of our knowledge, this is the first report regarding the successful treatment of a patient with DACA 'kissing' aneurysms characterized by bilateral frontal lobes completely adhering to each other. The management of such aneurysms is challenging, as the space available for dissection is limited (6). Additionally, by reviewing the relevant literature, the etiology, imaging characteristics, misdiagnosis reasons, treatment modalities and prognosis of DACA were investigated in order to improve the diagnosis and treatment of this disease.

\section{Case report}

A 54-year-old female patient was admitted to the People's Hospital of Hainan Province on January 2015 due to dizziness and headache accompanied by limb fatigue for $8 \mathrm{~h}$, without any evident disturbance of consciousness during the course of the disease. The patient had a previous history of hypertension for 2 years and had intermittently taken antihypertensive drugs on her own (details were not clear). In the outpatient department, a head computed tomography (CT) scan revealed an increased density in the interhemispheric fissure, bilateral Sylvian and basal cisterns (Fig. 1A). The present study was approved by the Ethics Committee of Hainan General Hospital (Hainan, China) and informed consent was received from the patient prior to the study.

Physical examination at admission revealed a blood pressure of 170/95 mmHg, Hunt-Hess scale II, normal consciousness, extremities muscle force grade 5, normal muscular tension, reduced superficial sensibility, equal and round pupils of $\sim 3 \mathrm{~mm}$ in diameter and reactive to light, and neck rigidity. Admission 
diagnosis was spontaneous subarachnoid hemorrhage with high probability of intracranial aneurysm rupture. Emergency cerebral digital subtraction angiography (DSA) indicated small aneurysms at segment A3 of bilateral anterior cereal arteries, $\sim 2 \times 2 \mathrm{~mm}^{2}$ with wide necks (Fig. 1B-D). Synthetic analysis of CT and DSA results did not determine the offending aneurysm; therefore, bilateral aneurysms should be treated together as otherwise there would be a great risk for another hemorrhage. Bilateral aneurysms were located at segment A3, the junction of pericallosal and callosomarginal arteries, with a small body size and wide neck. Furthermore, the aneurysm-bearing artery formed a right angle. Finally, microsurgical treatment was preferentially adopted using an interhemispheric fissure approach for aneurysm clipping.

The patient was immediately transferred to the operating room for emergency tracheal intubation following DSA examination. During treatment of the patient, as the longitudinal fissure was narrow, the surgical space was limited; therefore, it was likely to lead to aneurysm rupture during parent artery isolation. Conventional application of mannitol (20\% mannitol, 125 ml, 0.5 mg/kg, i.v., Hunan Kelun Pharmaceutical Co., Ltd., Yueyang, China) was performed as soon as the dura was cut, with anesthesiologists providing hyperventilation. Following satisfactory general anesthesia, an incision was made at the right forehead flap, and the forehead and bone flaps were successively freed. The dural tension was high and following detachment of the dura mater, the intracranial tension was also increased.

Next, the lateral cisterns were cut open to drain the cerebrospinal fluid (CSF) as much as possible, and the intracranial pressure was evidently decreased. Through longitudinal fissure, it was revealed that the bilateral frontal lobes completely adhered to each other from below the falx cerebri. As the bilateral frontal lobes grew together from the falx below the brain, the anterior communicating artery was detected making full use of the normal anatomical space. An incision was made into the anterior cranial base to expose the anterior communicating artery. Exploration was performed along the parent artery to the distal dissection of bilateral frontal lobes initiated from the segment of A2, and when hematoma was detected, which was likely to be the aneurysm area, separation of aneurysms was undertaken following temporary occlusion of bilateral aneurysms. Subsequently, an incision was made to dissect bilateral frontal lobes and to explore the bilateral anterior cerebral arteries to segment A3. The bilateral aneurysms were located at the bifurcation of segment A3, both $2 \times 2 \mathrm{~mm}^{2}$, and their bodies adhered to each other. Following dissection to thoroughly expose the aneurysm necks, one Yasagier 720 aneurysm clip and one Yasagier 740 aneurysm clip (Rebstock Instruments GmbH Dürbheim, Germany) were used to clip aneurysm necks. The aneurysms did not rupture and no hemorrhage occurred during clipping.

Following examinations performed to ensure that the aneurysms were completely clipped and the aneurysm-bearing arteries were open, the skull was closed. Due to the poor brain distal compensatory circulation, it was recommended that the blocking time should be $<10 \mathrm{~min}$ in order to avoid new nerve dysfunction following postoperative cerebral infarction, and the surgery was successful. After the patient woke up from the anesthesia, head CT was performed to exclude hemorrhage at the surgery areas (Fig. 2A). Furthermore, lumbar puncture was performed intermittently to drain CSF following the surgery. In total, 10 days later, re-examination demonstrated completely clipped aneurysms and fluent blood flow in aneurysm-bearing arteries (Fig. 2B-D). The patient recovered well and was discharged 12 days postoperatively with a Modified Rankin Scale $=0$ (7). A follow-up at 6 months after discharge was used to determine that the patient had recovered successfully.

Literature review. For the literature review, all articles referring to 'kissing aneurysms' in PubMed (https://www.ncbi.nlm.nih .gov/pubmed) were retrieved, and a total of 21 articles were a hit, of which one was an original report and remaining 20 were case reports. Altogether there were 30 cases of 'kissing' aneurysms reported in these articles (Table I). There were 12 male patients and 18 female patients, with a mean age of 46.4 years. In 4 cases, aneurysms were located at DACAs.

\section{Discussion}

Aneurysms of the DACA make up 3-7\% of intracranial aneurysms (6). As particular types of multiple aneurysms, 'kissing' aneurysms account for $<1 \%$ of intracranial aneurysms (8), and are most commonly observed at the supraclinoid internal carotid artery (this occurred in 15 cases in total from the literature reviewed in the present study, accounting for $\sim 50 \%$ of all cases). Furthermore, 'kissing' aneurysms at the DACA are rare, and there are a total of 5 known cases, including the present one. Analyses of previous case reports demonstrated that the morbidity of 'kissing' aneurysms is higher in females than in males (9), with no difference in the age of onset compared to other types of aneurysms. A study by Harada et al (10) classified 'kissing' aneurysms into two types according to whether their necks originated from the same aneurysm-bearing artery: Type I from the same artery and type II from different arteries. A study by Jagetia et al (11) reported a special type of 'kissing' aneurysms, which were located at a variant and fenestrated artery and could be classified as a sub-type of type I. Although a fenestrated artery has a normal morphology, the endothelium is thin, the elastic layer is discontinuous and the incidence of 'kissing' aneurysms is high (1). This indicates that the occurrence of 'kissing' aneurysms may be associated with congenital abnormality of the embryo (1,12). Among 5 cases of 'kissing' aneurysms reported by Jefferson (1), the first-grade relatives of 2 young patients had a history of aneurysms, supporting the viewpoint that this type of aneurysm may be affected by hereditary factors.

Furthermore, 'kissing' aneurysms are also associated with vascular structural changes, such as the anterior communicating artery complex $(4,13)$. Vascular structural changes may induce the formation of aneurysms via influencing the hemodynamics, although certain researchers doubt the reason of hemodynamics (2). In the present study, there were no evident abnormalities in the morphology and running of cerebral arteries. However, bilateral frontal lobes were adhered to each other, indicating the abnormal development of brain tissues, which may have interfered with the development of the aneurysm-bearing artery wall. In the present case, bilateral anterior cerebral arteries almost formed a right angle from segment A3 


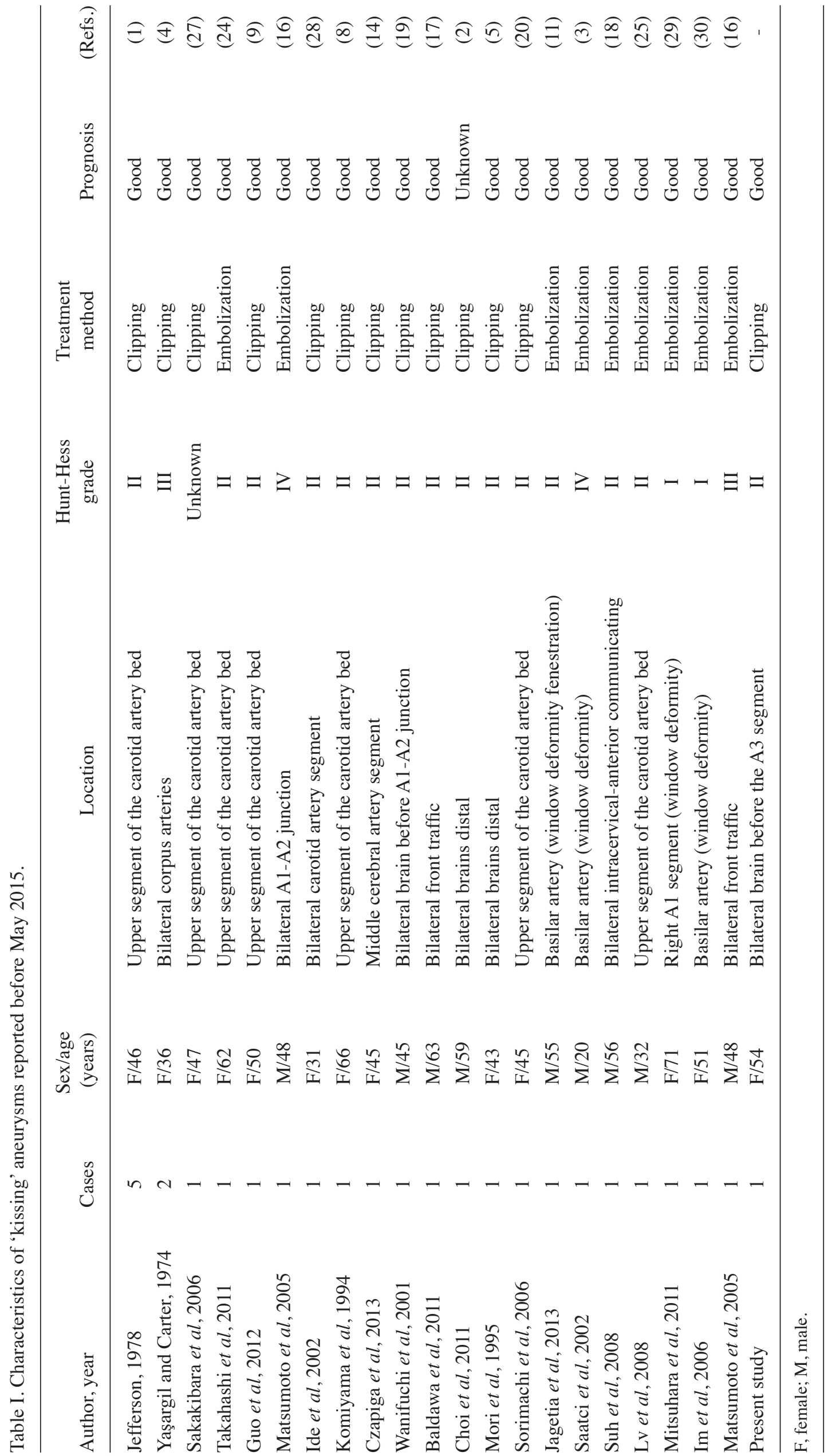



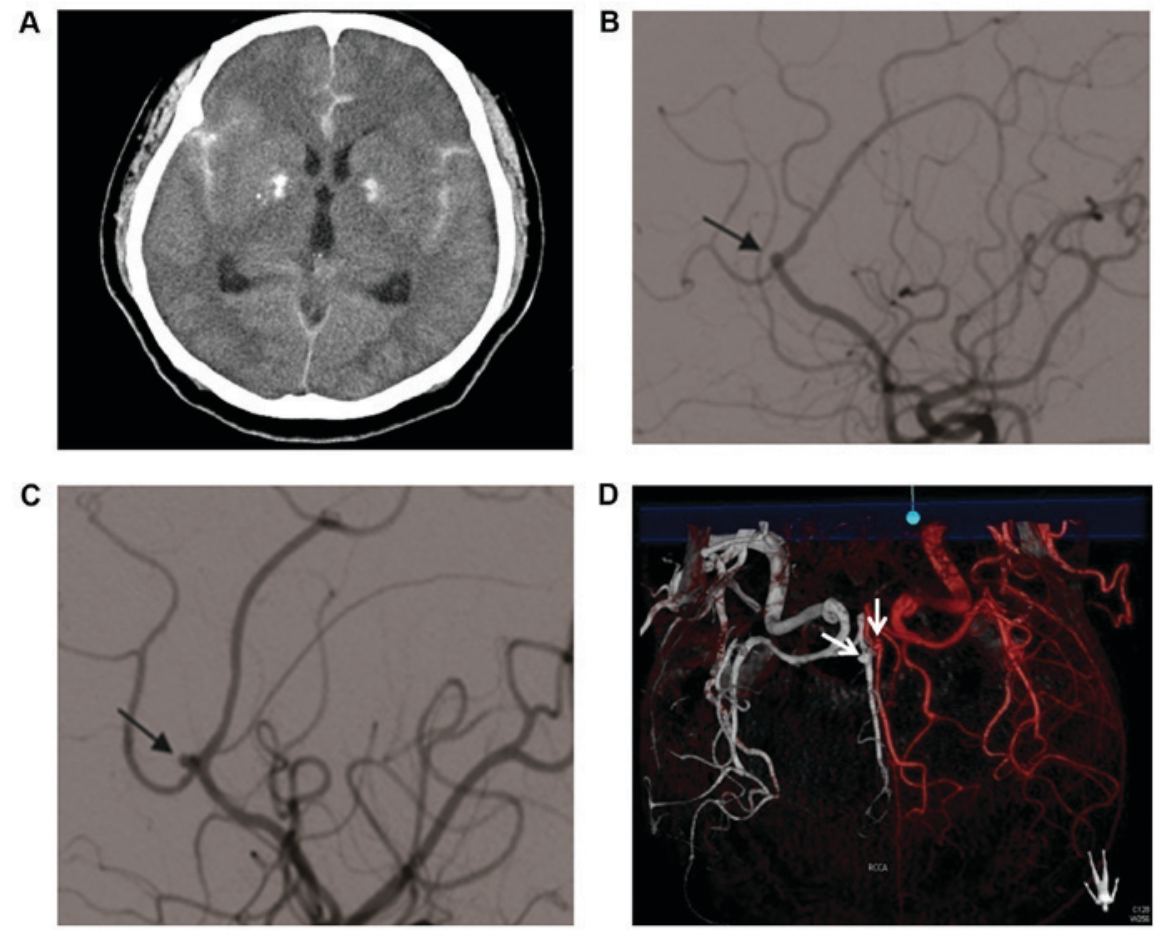

Figure 1. Patient preoperative carotid artery angiography, DSA and CT results. (A) Preoperative CT examination revealed subarachnoid hemorrhage. (B) The aneurysms of the A3 segment were observed on the left lateral carotid arteriography (black arrow). (C) Preoperative right internal carotid angiography lateral radiography also revealed A3 segment aneurysms (black arrow). (D) Preoperative three-dimensional DSA revealed bilateral A3 segment aneurysms that were adjacent (white arrows). DSA, digital subtraction angiography; CT, computed tomography.
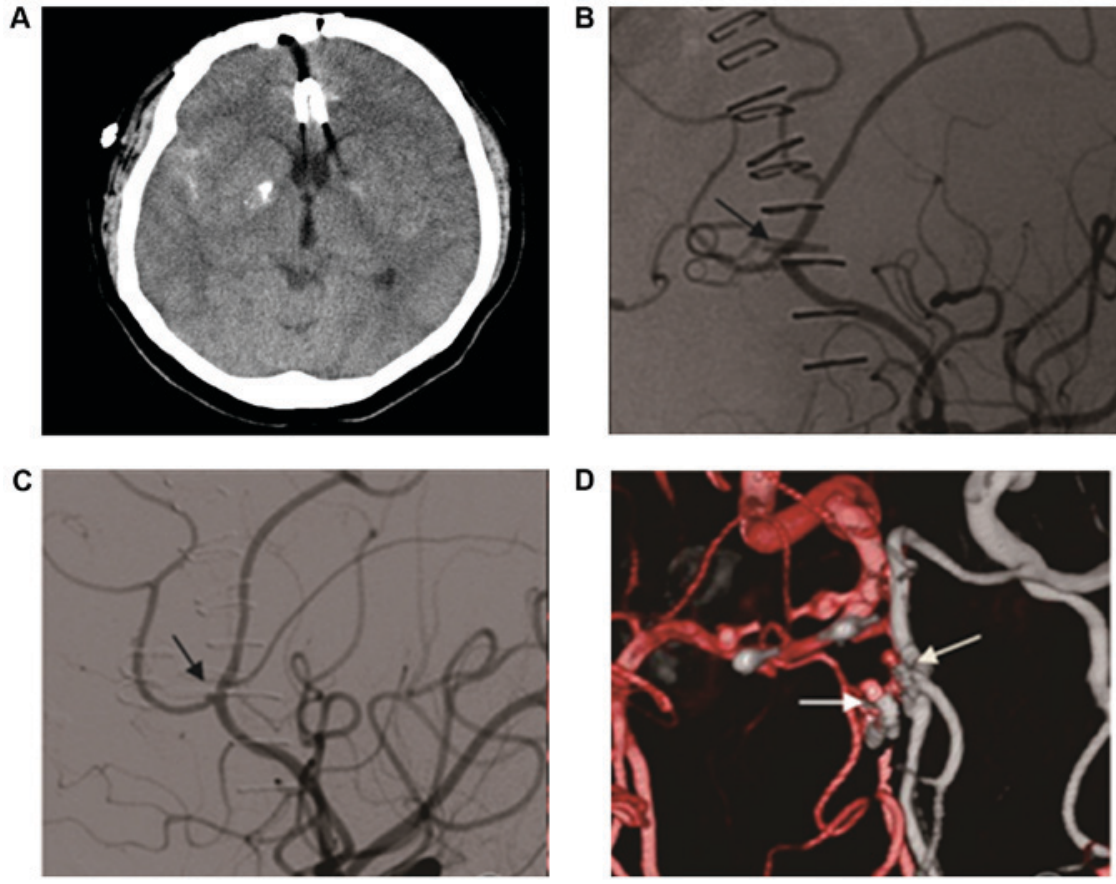

Figure 2. Patient postoperative carotid artery angiography, DSA and CT results. (A) High-density shadow for the aneurysm clip was depicted by postoperative CT, indicating that subarachnoid hemorrhage was reduced. Postoperative left (B) and right (C) lateral carotid artery angiography demonstrated that the left A3 segment aneurysm completely disappeared. The black arrow indicates the position where the segment aneurysm was located. (D) Three-dimensional DSA images of the left and right internal carotid arteries following surgery indicated that the segment aneurysm had completely disappeared. The white arrow indicated the position where the segment aneurysm was located. DSA, digital subtraction angiography; CT, computed tomography.

to A4, and were subjected to a strong impact force from the blood flow. The above two factors contributed to the formation of aneurysms.
The clinical manifestations of 'kissing' aneurysms are similar to other types of aneurysms, they mainly manifest with a subarachnoid hemorrhage and space occupying effect (14). 
There is only 1 case of ventricle hematocele not accompanied by intracranial hematoma (15). The specific structure of partial fusion of aneurysm walls makes diagnosis difficult, and they are often misdiagnosed as a single irregular or lobulated aneurysm (16). Statistical analysis by Harada et al (10) demonstrated that $57 \%$ of 'kissing' aneurysms had been misdiagnosed prior to surgery. Precise preoperative diagnosis of 'kissing' aneurysms is paramount, and it may be improved in the following aspects. First, 'kissing' aneurysms are common in the internal carotid, bilateral posterior communicating, anterior communicating and anterior cerebral arteries, with different neck locations. Dynamic observation during DSA may find that 'kissing' aneurysms of the same aneurysm-bearing artery have a prolonged filling phase. For suspected pulmonary aneurysms, adding multi-angle oblique scanning and capturing compression images may make a differential diagnosis. Second, three-dimensional (3D)-DSA and angiography at a specific angle (17) or 3D-CT angiography is helpful for diagnosis. It is quite intriguing to determine the offending aneurysm for 'kissing' aneurysm hemorrhage due to a lack of reported cases $(2,16)$ and limited literature available for reference. Generally, a diagnosis could be made in accordance with the determination of the offending aneurysm of multiple aneurysms: Irregular aneurysm wall with small vesicle-like bulges and the transparent blue aneurysm wall occasionally attached with blood clot identified during surgery (9). In the present case, the right aneurysm was more irregular than the left and was diagnosed as the offending aneurysm prior to the surgery, which was confirmed during the surgery.

In the present study, the 'kissing' aneurysms were thought to be adjacent, therefore, the two aneurysms could be clipped through one surgery route, and it would be easy to determine the offending aneurysm in order to explore the aneurysm-bearing artery and to emergently and temporarily occlude it during the surgery. If interventional embolization was feasible, two sets of micro catheters could be used to embolize bilateral aneurysms simultaneously or to prevent aneurysm rupture during the surgery. Therefore, compared with intracranial multiple aneurysms located far from each other, it is not imminent to determine the offending aneurysm of 'kissing' aneurysms.

A literature review revealed that the previous 3 cases of 'kissing' aneurysms at the anterior cerebral arteries were all treated with microsurgery of aneurysm clipping $(2,4,5)$. In the present case, it was difficult to control the catheter in the distal portion of the artery and bilateral aneurysms were small with wide necks, making complete embolism impossible. Additionally, a spring coil (a detachable spiral metallic material used for embolization of an aneurysm by an arterial approach) may have migrated and caused occlusion of the DACA, leading to a large area of cerebral infarction in the functional areas of the frontal lobe. Aneurysms were located at segment A3 at the bifurcation of pericallosal and callosomarginal arteries, where a right angle was formed and artery lumen was narrow, making it difficult to transport and place the stent. With reference to literature and by weighing the pros and cons, bilateral aneurysm clipping was performed through the interhemispheric fissure approach subsequent to opening the skull in the right forehead $(2,5)$.

The aneurysms at the segment A3 of the anterior cerebral arteries were located deep in the longitudinal fissure.
The narrow interhemispheric space and cerebral edema following subarachnoid hemorrhage left little room for surgery. Additionally, arachnoid adhesion and occlusion of a number of cisterns made it difficult to open the cistern for CSF drainage. In order to expose aneurysm necks, it may be necessary to sacrifice the bridging veins, which could exacerbate postoperative cerebral edema (17). In the present study, the aneurysms were partly attached to the frontal lobes, thus they may have ruptured again during brain tissue stretching or aneurysm isolation during the surgery. A study by Suh et al (18) reported an intra-operative rupture rate of $50 \%$ during surgery on DACA aneurysms, which was much higher than that of aneurysms from other sites. Intra-operative CSF drainage to reduce intracranial pressure and prevent aneurysm rupture is pivotal for successful therapy (17). One peculiar aspect of the present case is that the patient's bilateral frontal lobes were completely adhered to each other from below the falx cerebri during surgery, which markedly increased the difficulty in exposing aneurysms and the risk of aneurysm rupture. Thorough preparations were prepared for this situation, such as the administered of mannitol for dehydration to reduce intracranial pressure along with skull opening. Arachnoid membrane was carefully and sharply dissected to drain CSF from the interhemispheric fissure cistern during surgery. After achieving satisfactory intracranial pressure, bilateral segment A1 of the anterior cerebral arteries and anterior communicating arteries were dissected and exposed through the anterior cranial fossa. Subsequently, frontal basal tissues were cut open longitudinally along the anterior cerebral arteries, and an upward exploration to the bilateral aneurysms at segment A3 was performed, during which time we took the advantage of the temporary occlusion technique (9), and clipped the bilateral aneurysms parallel to the anterior cerebral arteries following careful isolation of the aneurysm necks. Subsequently, puncture examination was performed to check whether aneurysms were completely clipped. An attempt was made not to dissect the adhesion walls between aneurysms prior to clipping to prevent rupture and hemorrhage $(19,20)$. The advantage of this method is that once aneurysm rupture and hemorrhage occur in the course of exploration for aneurysms, they may be instantly and temporarily blocked. The limitation is that a relatively large area of injured brain tissues may exacerbate postoperative cerebral edema and cause postoperative hemorrhage in the surgery area. Previously, a number of researchers have used the navigation system (21) and intraoperative ultrasound (22) to pinpoint intraoperative brain tissue injury during anterior cerebral artery aneurysm surgery. These techniques may also be applied in the surgery on 'kissing' aneurysms of DACA.

Compared with microsurgery, interventional embolization causes less injury to the nerve tissues and reduces the incidence of complications. Furthermore, it should be a preferential therapy (6), particularly for patients with poor general conditions (23). Researchers have treated aneurysms with interventional embolization at the vertebrobasilar, middle cerebral, bilateral ophthalmic and anterior communicating arteries with favorable therapeutic effects $(11,18,24,25)$. Although interventional embolization for the treatment of distal anterior cerebral aneurysms has been reported to be beneficial $(13,26)$, thus far reports of this therapy on 'kissing' aneurysms of DACA are rare. The possible reasons are as 
follows: A number of cases of 'kissing' aneurysms at DACA were treated with interventional embolization and could not be diagnosed by imaging and their diagnosis was missed $(10,16)$. Furthermore, the long distance and narrow lumen of DACA make it difficult for micro-catheter manipulation (18).

'Kissing' aneurysms at DACA are mostly small, thus the walls are easily punctured during micro-catheter and guided-wire manipulation, causing hemorrhage, which is hard to repair (2). Furthermore, a wide neck is commonly observed and the aneurysm-bearing artery is evidently curved, making it difficult to implant the stent (9). The structure of 'kissing' aneurysms are complex, and it is hard for surgeons to grasp their complete morphology, resulting in an increase in unpredictable factors during the surgery. Furthermore, familiarity of surgeons with the two therapies may also influence their choice of surgery strategies. By accumulating the experience of endovascular treatment and improvement in interventional materials, 'kissing' aneurysms of DACA may be well treated with embolization.

The patient of the present study recovered well and was discharged without defects in neurological functions. In previous reports, the majority of patients with 'kissing' aneurysms had a good prognosis, with only one case of unknown prognosis, reaching a good prognosis rate of $96.8 \%$. This may predominantly be attributed to the following factors: i) The majority of the patients had a good pre-operative condition, and only 4 of them had high Hunt-Hess scales; ii) some 'kissing' aneurysms patients underwent interventional embolization, which made it impossible to identify whether the aneurysms had partially adherent walls. As a result, this lead to missed diagnosis and affected the prognosis analysis; and iii) the sample size was not large enough, and there were too few cases to thoroughly demonstrate a complete prognosis of 'kissing' aneurysms.

In conclusion, DACA is easily misdiagnosed and the presence of bi-lobed aneurysms indicated the presence of kissing aneurysms. Further examination of 3D DSA is helpful for diagnosis and the application of the longitudinal fissure approach to block the distal anterior cerebral artery is conducive to DACA.

\section{References}

1. Jefferson A: The significance for diagnosis and for surgical technique of multiple aneurysms of the same internal carotid artery. Acta Neurochir (Wien) 41: 23-37, 1978.

2. Choi CY, Han SR, Yee GT and Lee CH: Kissing aneurysms of the distal anterior cerebral artery. J Clin Neurosci 18: 260-262, 2011.

3. Saatci I, Cekirge HS, Karcaaltincaba M, Basgun N, Berker M, Timurkaynak E and Ozcan OE: Endovascular treatment of kissing aneurysms at the fenestrated basilar artery. Case report with literature review. Surg Neurol 58: 54-58, 2002.

4. Yaşargil MG and Carter LP: Saccular aneurysms of the distal anterior cerebral artery. J Neurosurg 40: 218-223, 1974.

5. Mori T, Fujimoto M, Shimada K, Shin H, Sakakibara T and Yamaki T: Kissing aneurysms of distal anterior cerebral arteries demonstrated by magnetic resonance angiography. Surg Neurol 43: 497-499, 1995.

6. Otani N, Takasato Y, Masaoka H, Hayakawa T, Yoshino Y, Yatsushige H, Sumiyoshi K, Miyawaki H, Aoyagi C, Takeuchi S and Suzuki G: Clinical features and surgical outcomes of ruptured distal anterior cerebral artery aneurysms in 20 consecutively managed patients. J Clin Neurosci 16: 802-806, 2009.
7. Banks JL and Marotta CA: Outcomes validity and reliability of the modified Rankin scale: Implications for stroke clinical trials: A literature review and synthesis. Stroke 38: 1091-1096, 2007.

8. Komiyama M, Yasui T, Tamura K, Nagata Y, Fu Y and Yagura H: 'Kissing aneurysms' of the internal carotid artery. Neurol Med Chir (Tokyo) 34: 360-364, 1994.

9. Guo L, Qiu Y, Ge J and Zhang X: Kissing aneurysms of the internal carotid artery treated with surgical clipping. Neurol India 60: 353-355, 2012.

10. Harada K, Orita T and Ueda Y: Large kissing aneurysms of the middle cerebral artery: A case report-classification of kissing aneurysms. No Shinkei Geka 32: 513-517, 2004.

11. Jagetia A, Bhutte M, Aher R, Sinha S and Singh D: Kissing aneurysm in a fenestrated mid-basilar arterial trunk: A case report and review of literature. Neurol India 61: 437-439, 2013.

12. Krings T, Baccin CE, Alvarez H, Ozanne A, Stracke P and Lasjaunias PL: Segmental unfused basilar artery with kissing aneurysms: Report of three cases and literature review. Acta Neurochir (Wien) 149: 567-574, 2007.

13. Enesi E, Rroji A, Demneri M, Vreto G and Petrela M: Mirror image distal anterior cerebral artery aneurysms treated with coil embolization. a report of two cases and literature review. Interv Neuroradiol 19: 49-55, 2013.

14. Czapiga B, Kozba-Gosztyla M, Bereza S and Jarmundowicz W: Unusual angiographic images of unruptured, large, kissing middle cerebral artery aneurysms. Acta Neurochir (Wien) 155: 283-284, 2013.

15. Fujimura M, Sugawara T, Higuchi H, Oku T and Seki H: A ruptured aneurysm at the distal end of the basilar artery fenestration associated with multiple fenestrations of the vertebrobasilar system: Case report. Surg Neurol 47: 469-472, 1997.

16. Matsumoto H, Takechi A, Kohno K and Sasaki U: 'Kissing aneurysms' of the anterior communicating artery treated with coil embolization. J Endovasc Ther 12: 750-754, 2005.

17. Baldawa SS, Menon G and Nair S: Kissing anterior communicating artery aneurysms: Diagnostic dilemma and management issues. J Postgrad Med 57: 44-47, 2011.

18. Suh SJ, Kang DG, Ryu KY and Cho JH: Endovascular treatment of 'kissing aneurysms' at the anterior communicating artery. J Korean Neurosurg Soc 44: 163-165, 2008.

19. Wanifuchi H, Shimizu T, Higa T and Nakaya K: Kissing mirror image anterior communicating artery aneurysms-case report. Neurol Med Chir (Tokyo) 41: 29-32, 2001.

20. Sorimachi T, Fujii Y, Nashimoto T and Morita K: Kissing aneurysms at the junction of the internal carotid artery and the ipsilateral duplicate anterior choroidal arteries-case report. Neurol Med Chir (Tokyo) 46: 29-31, 2006.

21. Zhang T, Li F and Zheng G: Application of neuronavigation in the surgery for distal anterior cerebral aneurysm. J China-Japan Friendship Hospital 27: 79-81, 2013 (In Chinese).

22. Piao J, Luo Q and Yu J: Surgical treatment of ruptured anterior cerebral artery aneurysm (22 cases report). Chin J Nerv Dis: 431-432, 2013 (In Chinese).

23. Park KY, Kim BM, Lim YC, Chung J, Kim DJ, Joo JY, Huh SK, Kim DI, Lee KC and Lee JW: The role of endovascular treatment for ruptured distal anterior cerebral artery aneurysms: Comparison with microsurgical clipping. J Neuroimaging 25: 81-86, 2015.

24. Takahashi C, Kubo M, Okamoto S, Matsumura N, Horie Y, Hayashi N, Kuwayama N and Endo S: 'Kissing' aneurysms of the internal carotid artery treated by coil embolization. Neurol Med Chir (Tokyo) 51: 653-656, 2011.

25. Lv X, Li Y and Wu Z: Endovascular management for bilateral ophthalmic segment 'kissing' aneurysms presenting with nasal bleeding. A case report. Neuroradiol J 21: 266-269, 2008.

26. Oishi H, Nonaka S, Yamamoto M and Arai H: Feasibility and efficacy of endovascular therapy for ruptured distal anterior cerebral artery aneurysms. Neurol Med Chir (Tokyo) 53: 304-309, 2013. 